\title{
Wearable Diagnostics - A Brief Outlook, Prospects and Future
}

\section{Sheela B and Subbiah $A^{*}$}

CSIR-Central Electrochemical Research Institute, Karaikudi, Tamilnadu, India

We live in an era where service sectors advertise their ability to perform their activities "online" and about running paperless transactions. This trend is most evident in the healthcare sector for online monitoring of an individual's health status at home or at travel, by incorporating flexible/body worn diagnostic and implantable devices attached to the body. Further, web enabled meetings and conferences with the medical practitioners exclude our travel and ambulatory requirements. The requirement for wearable diagnostics stems from the need for monitoring patients over long periods of time. These devices are non-invasive, non-obtrusive and have the potential to continuously collect vital health information from a person's body and transmit the data either to the individual's concerned (doctors/nurses) or to their healthcare provider on time. Such devices can send alerts regarding any imminent health hazard to provide solutions at the right time outside the hospital environment. This would be especially helpful for monitoring senior citizens or patients at home suffering from chronic diseases (particularly in remote locations where they have no personal access or limited access to doctors). Data transmission can be enabled through smart phones which provide a highly convenient platform for constant monitoring of signals from a device. These developments hold considerable promise for maintaining and improving the quality of life at affordable medical costs. Most of the efforts in the development of wearable diagnostics have been devoted to the continuous monitoring of parameters such as heart rate, respiration rate and skin temperature. However, wearable chemical sensors in the form of bandage or smart shirts need to be developed at a faster pace to fill the gaps in wearable sensor technology. The two important aspects during the design of wearable technology will be light-weight and durability. Wearable diagnostics enables on-site health management platforms in every individual. Body worn devices to measure disease markers in saliva and sweat are emerging rapidly. Efforts are also put forth to fabricate wearable energy harvesting technology in order to power the wearable devices. The possibility of miniaturization of electronic components, nanolithography techniques and smart textiles have resulted in the design of new class of "wear and forget" type sensors without compromising the comfort of the wearer. With rapid advancements in the printing process, transduction techniques and printed electronics, the textile based wearable sensors will find a unique place in medical diagnostics.
*Corresponding author: Subbiah A, Senior Scientist, Bio-Electrochemistry Group, CSIR-Central Electrochemical Research Institute (Govt of India), Karaikudi, 630006, Tamilnadu, India, Tel no: +91-4565-241454 (Off); Fax: +91-4565-227713 (Off); E-mail: salwarap@gmail.com; alwarappan@cecri.res.in

Received May 18, 2015; Accepted May 20, 2015; Published June 01, 2015

Citation: Sheela B, Subbiah A (2015) Wearable Diagnostics - A Brief Outlook, Prospects and Future. J Biosens Bioelectron 6: e138. doi:10.4172/2155$6210.1000 \mathrm{e} 138$

Copyright: ( $) 2015$ Sheela B, et al. This is an open-access article distributed under the terms of the Creative Commons Attribution License, which permits unrestricted use, distribution, and reproduction in any medium, provided the original author and source are credited. 